\title{
La gestion de projet basée sur la stratégie organisationnelle ${ }^{1}$
}

\author{
Yuhong Beiyu \\ Zhejiang Gongshang University, Chine
}

\section{Introduction}

$\mathcal{A}$ vec l'adhésion de la Chine à l'organisation mondiale du commerce (OMC), de plus en plus d'entrepreneurs réalisent que l'arrimage entre gestion stratégique et gestion de projet est essentiel à leur survie face aux transformations du marché mondial. Mais les rôles respectifs que jouent la gestion stratégique et la gestion de projet sont souvent mal compris. Le but ultime de la gestion stratégique organisationnelle est de donner une direction générale au plan stratégique d'une entreprise pour lui permettre de survivre à la concurrence féroce du marché. Des stratégies organisationnelles basées sur des analyses scientifiques sont mises en place afin de réagir promptement à un environnement de marché qui change constamment.

En adaptant son système

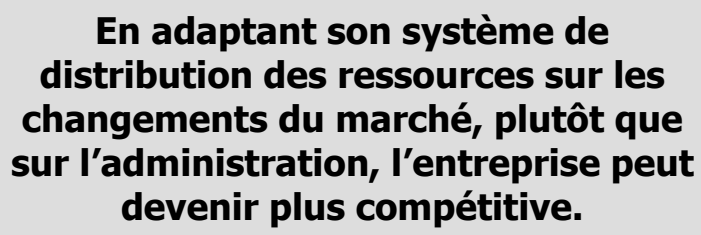

En adaptant son système de distribution des ressources sur les changements du marché, plutôt que sur l'administration, l'entreprise peut devenir plus compétitive.

La gestion stratégique organisationnelle est à prime abord un processus par lequel les dirigeants de l'entreprise doivent décider: «Qui sommes-nous?» «Quel est notre objectif? " «Que doit-on faire pour y arriver? ». La gestion de projet comprend quatre phases : l'analyse stratégique, le choix stratégique, l'application stratégique et l'évaluation-contrôle stratégique (figure 1).

La raison d'être de l'analyse stratégique est d'évaluer les facteurs décisifs qui affectent le développement présent et futur d'une organisation ainsi que l'identification des facteurs qui influencent la mise en œuvre de la stratégie choisie.

Les trois aspects principaux sont : 1) la détermination de l'objectif orgade distribution des ressources sur les changements du marché, plutôt que sur l'administration, l'entreprise peut devenir plus compétitive. Cependant, une méthodologie applicable et efficace s'impose. C'est là que la gestion de projet entre en jeu. Celle-ci peut aider les entreprises modernes à mettre sur pied une gestion systématique et efficace en ce qui a trait au produit à distribuer, à la gestion du service qui entoure le produit et aux modifications organisationnelles rencontrées en cours de route.

La gestion de projet repose sur le travail coopératif, donc sur la division des tâches. Par le passé, même un projet bien choisi et porté à terme pouvait rater l'objectif stratégique de l'organisation. C'est la raison pour laquelle de plus en plus d'entreprises essaient maintenant d'allier gestion stratégique à gestion de projet dans leur planification stratégique globale. nisationnel, 2) l'analyse de l'environnement externe et 3) l'analyse de la condition interne.

L'analyse stratégique vise à définir la position actuelle de l'entreprise face au marché, mais aussi à faire l'inventaire de ses ressources et de ses compétences stratégiques. Elle vise également à prédire ce à quoi s'attendent les actionnaires, comment ceux-ci vont réagir à la façon dont la stratégie est menée et finalement, quel effet aura la réaction des actionnaires sur le rendement de l'entreprise par rapport à l'objectif à atteindre.

L'analyse stratégique identifie le statut actuel de l'entreprise, alors que le choix stratégique se penche sur les façons et les moyens concrets d'atteindre l'objectif stratégique. 


\section{Figure 1 - Gestion stratégique organisationnelle}

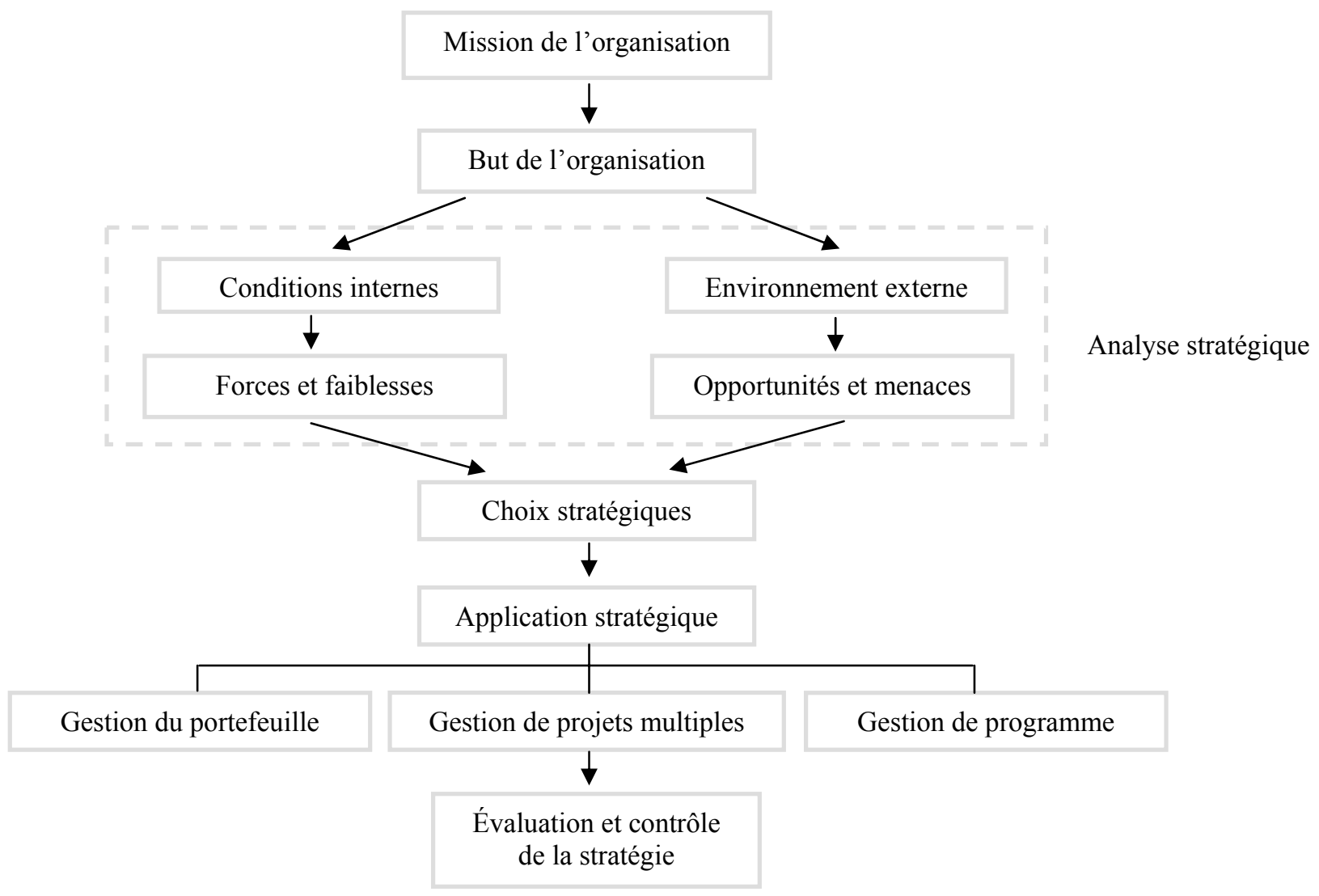

L'application de la stratégie organisationnelle de l'entreprise doit répondre aux questions suivantes :

- Comment les ressources disponibles doivent-elles être distribuées et utilisées à travers les différents départements d'une entreprise?

- Quelles sont les ressources externes qui doivent être mises à contribution afin que l'objectif stratégique soit atteint?

- Quels ajustements devront être apportés pour améliorer la structure de l'entreprise?

- Comment faire face aux problèmes de la distribution du produit?

- Comment s'assurer que la stratégie choisie soit compatible avec la vocation de l'entreprise ?

Le but de l'évaluation et du contrôle de la stratégie est d'en estimer le succès final, mais aussi d'évaluer les différentes étapes de son application.
L'étape la plus difficile à mettre en application est celle de la stratégie. Dans la pratique de gestion, il est démontré que choisir une stratégie occupe environ $20 \%$ du travail, alors que la mise en place en occupe $80 \%$. Il n'est pas suffisant de planifier la stratégie, encore faut-il voir à sa bonne exécution.

\section{La gestion de projet organisationnelle}

La gestion de projet vise la réussite d'un projet, malgré les contraintes et le manque de ressources. Elle couvre la réalisation complète du projet, de la décision initiale jusqu'à sa conclusion finale, en passant par les différentes phases de la planification, de l'organisation, de la supervision, de la coordination, du contrôle et finalement de l'évaluation. La gestion de projet est partagée entre sept grandes aires de compétence :

- gestion de l'horaire du projet;

- gestion du coût;

- gestion de la qualité; 
- gestion des ressources humaines;

- gestion des communications;

- gestion des risques;

- gestion des achats liés au projet.

Auparavant, la gestion de projet reposait principalement sur le projet lui-même. Les gestionnaires abordaient la gestion de projet à l'aide de techniques pourtant éprouvées telles que PERT/CPM ${ }^{2}$ et $\mathrm{WBS}^{3}$. Les résultats étaient parfois couronnés de succès, mais ils étaient souvent désastreux. L'approche globale et systématique ne faisait alors pas partie de l'élaboration de la gestion de projet, écartant ainsi toute stratégie organisationnelle. Sans l'aide d'un système de priorité de projet, ou sans une méthode de classement de projets par priorité de ressources, il est impossible d'identifier les projets qui sont les plus aptes à contribuer au plan stratégique. Dans un tel contexte, les outils et techniques utilisés perdent leur valeur utile. L'effort déployé sur la réalisation de projets individuels peut donner des résultats positifs, mais souvent au détriment de l'objectif à long terme de l'entreprise. En l'absence d'une vision globale, les projets individuels peuvent même se nuire entre eux. Une lacune au plan du contrôle à long terme peut conduire à un échec de la gestion de projet. La gestion de projet doit donc être abordée d'une façon différente, qui tienne compte du plan stratégique à long terme de l'entreprise. L'entreprise de gestion de projet (EGP) est donc appelée à devenir la nouvelle tendance dans la gestion organisationnelle.

Le principe de base de l'EGP repose sur la gestion de tous les projets d'une entreprise par rapport à son objectif stratégique global. Une stratégie de gestion est d'abord mise sur pied. Celle-ci comprend trois sous-systèmes : la gestion de projets multiples, la gestion de portefeuille et la gestion de programme.

EGP est synonyme de gestion organisationnelle à long terme. Dans cette optique, la gestion de projet fait passer l'orientation de la gestion organisationnelle à long terme de « base fonctionnelle » à « base cible ».

\section{Figure 2 - Relation entre l'EPG et la stratégie organisationnelle}

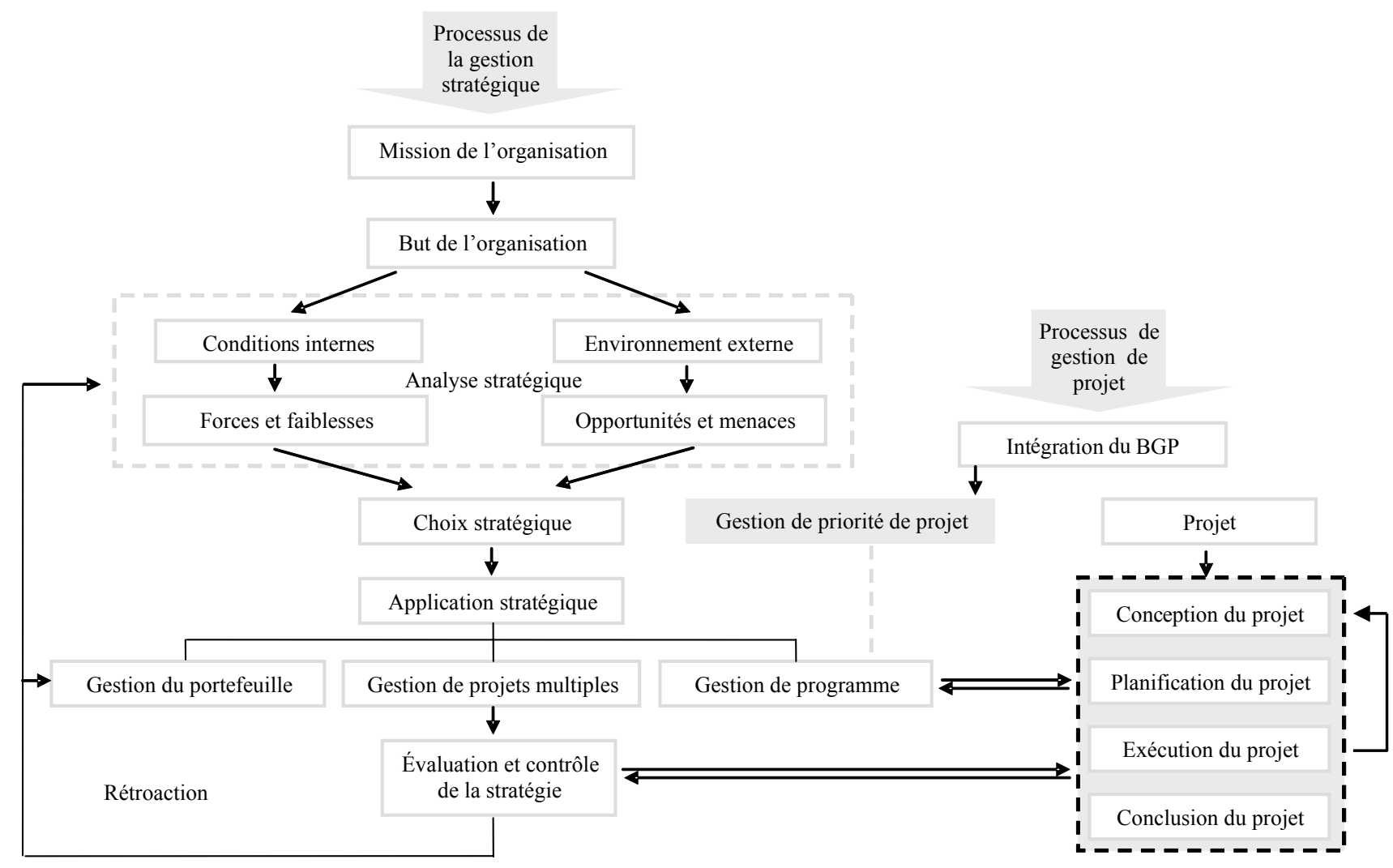


La figure 2 illustre la relation entre l'EPG et la stratégie organisationnelle. L'EPG est plus que de la gestion de projets indépendants. Ce type de gestion permet l'instauration systématique d'un modèle intégré à la gestion de tous les projets en cours. Ce modèle permet non seulement à la gestion de projet de jouer un rôle fondamental dans la gestion stratégique de l'entreprise, il permet aussi d'en améliorer constamment le rendement. Autrement dit, ce modèle met l'emphase sur la visée stratégique d'une entreprise en traitant celle-ci comme une entité qui dépasse la simple réalisation d'un projet. Même un projet temporaire devient ainsi étroitement lié à la mission de l'entreprise, car tout projet n'est conçu que dans l'optique de l'atteinte de l'objectif final. L'utilisation du cadre de l'EPG au plan stratégique a un impact positif sur le système de gestion d'une entreprise, car tout repose désormais sur la réussite de l'objectif global et non sur le processus.

\section{Le bureau de gestion de projet (BGP)}

Afin de répondre aux différents besoins que nécessite la réalisation d'un projet, la mise en place de structures parallèles de soutien est vitale. Ces structures sont: l'organisation fonctionnelle, l'organisation de projet et l'organisation matricielle.

Le succès du système de gestion de projet repose sur le délicat équilibre qui existe entre les exigences du projet et celles de l'entreprise. C'est le rôle de l'organisation matricielle d'allier les besoins et les ressources de différents projets en cours à l'intérieur d'une même entreprise. L'organisation matricielle utilise les ressources de deux autres organisations : l'organisation fonctionnelle et l'organisation de projet. Mais si l'organisation matricielle est théoriquement idéale, elle demeure néanmoins difficile à mettre en pratique, car elle génère des problèmes de communication importants. C'est pourquoi un autre type de structure est nécessaire, d'où la création d'une gestion de projet centralisée ou d'un bureau de gestion de projet (BGP).

\section{Fonction du BGP dans la structure organisationnelle}

La place du BGP dans la structure organisationnelle est illustrée à la figure 3. À la tête du BGP se trouve un gérant général qui est autorisé à utiliser et à répartir toute ressource nécessaire aux différents départements sous sa charge. Le rôle du gérant général est de superviser, guider et évaluer le progrès des différents projets en cours.

Le BGP doit rester fidèle à la stratégie globale de l'entreprise, à sa mission et à son objectif. Même si plusieurs projets se déroulent simultanément et indépendamment les uns des autres, le BGP assure la cohésion de leur objectif stratégique commun, soit la compétitivité de l'entreprise au sens large.

\section{Figure 3 - La place du BPG dans la structure organisationnelle}

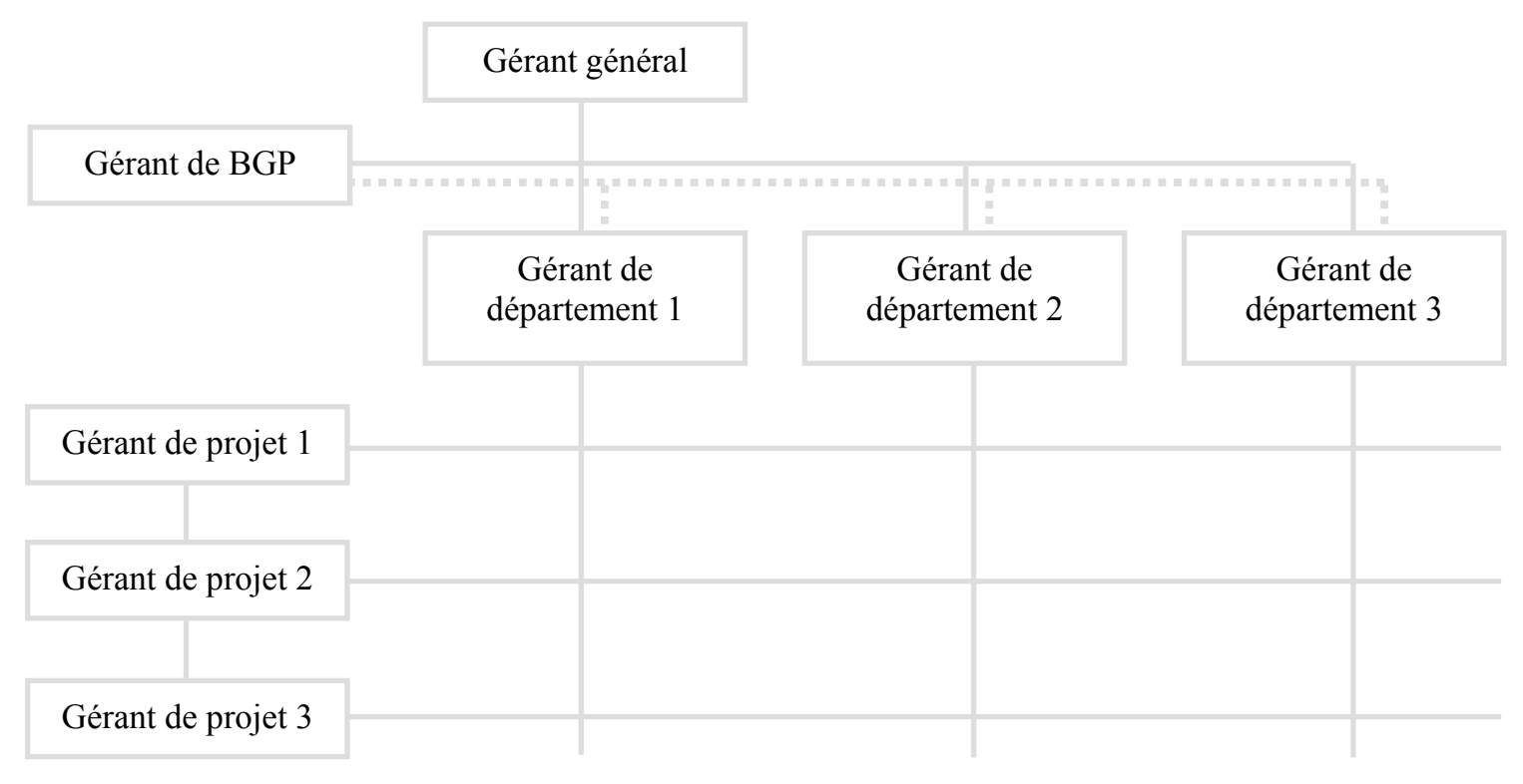




\section{Le processus de gestion de projet comprend quatre phases.}

\section{Fonction du BGP}

Le rôle principal du BGP est d'aider l'entreprise à se démarquer sur le marché. Cette aide comprend la gestion du portefeuille, l'élaboration des critères, les procédures de projet, la formation des gestionnaires de projet, la mise en place de la culture de projet et la logistique des besoins et ressources à mettre en place.

La gestion du portefeuille est ce qui est le plus important à l'intérieur du BGP et elle a deux tâches principales :

1. La validation de la stratégie de l'entreprise par rapport à sa mission et à son objectif ainsi que le choix et l'exécution de projets appropriés. Un lien serré doit être tissé entre tous les projets en cours afin que l'objectif global établi préalablement par la haute direction soit atteint.

2. La mise sur pied d'un système de priorité dans la sélection de projets. Un ordre de priorité est essentiel, car il existe toujours des restrictions qui influencent la réalisation d'un projet. Les projets retenus doivent non seulement suivre la ligne directrice de la stratégie organisationnelle de l'entreprise, ils doivent aussi être efficaces et économiques.

\section{Fusion de la gestion de stratégie organisationnelle et de la gestion de projet organisationnel}

Le bureau de gestion de projet joue un rôle vital dans l'implantation et l'intégration de ces deux types de gestion. Le processus de gestion de projet comprend quatre phases: le choix du projet, sa planification, l'exécution et la livraison du produit. Le processus de l'intégration de la gestion stratégique à celle de la gestion de projet se déroule de la façon suivante :

1. Un BGP est d'abord mis en place, ses fonctions et pouvoirs sont clairement définis.

2. Le BGP établit un système de priorité de projet qui tient compte de l'objectif global de l'entreprise. Le BGP s'assure également que le proces- sus de sélection des projets est libre de toute interférence liée à la politique ou aux jeux de pouvoir interne.

3. Le BGP fait en sorte que l'objectif des projets en cours ne déroge pas du plan stratégique de l'entreprise. La coopération entre les différents niveaux d'exécution est primordiale, de la haute direction au plus bas échelon de la division des tâches.

4. Seuls les projets réalistes doivent être envisagés. La décision de retenir ou non un projet repose sur la disponibilité des ressources, la logistique et les coûts.

5. Puisque l'application de la stratégie globale repose sur l'exécution et la réussite de différents projets menés simultanément, le contrôle et l'évaluation de la stratégie reviennent logiquement à la gestion de contrôle de projet.

La fusion de ces deux modes de gestion est donc appelée à modifier l'approche conventionnelle de l'atteinte de l'objectif en forçant la coopération et la coordination entre projets afin qu'ils soient orientés vers un but commun : la rentabilité de l'entreprise.

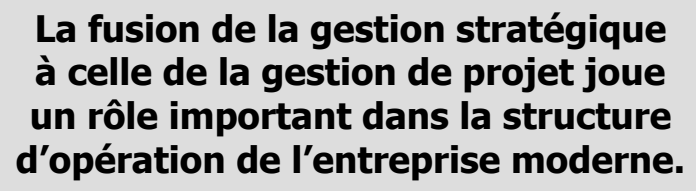

\section{Conclusion}

L'entreprise n'a d'autre choix que de s'adapter à un marché dont les demandes changent constamment. Afin de demeurer en position concurrentielle, elle doit développer une gestion stratégique souple et polyvalente, une gestion capable de s'ajuster rapidement et efficacement aux fluctuations soudaines du marché. La fusion de la gestion stratégique à celle de la gestion de projet joue un rôle important dans la structure d'opération de l'entreprise moderne. Ce nouveau mode de gestion contribue ainsi à placer l'entreprise dans une position concurrentielle favorable sur le marché mondial en facilitant le pouvoir décisionnel entre les projets, tout en priorisant la mission et l'objectif stratégique global de l'entreprise. 


\section{Notes et références}

1 La traduction de cet article de l'anglais au français a été réalisée par monsieur Richard Cloutier.

2 À la base, la CPM (Critical Path Method : méthode critique du cheminement) et le PERT (Program Evaluation Review Technique: révision technique d'évaluation de programme) sont des techniques de gestion de projet qui ont été créées pour servir les intérêts industriels et militaires du monde occidental.

Source : http://www.interventions.org/pertcpm.html.
3 Quand on parle de gestion de projet, la WBS (Work Breakdown Structure : structure de fragmentation des tâches) est un cadre exhaustif et hiérarchique des tâches à accomplir dans l'élaboration et la mise à terme d'un projet. La WBS est un outil de gestion essentiel. À tel point que plusieurs branches exécutives du gouvernement des États-Unis exigent qu'il fasse partie de leur structure organisationnelle. Source : Wikipedia, the free encyclopedia : http://en.wikipedia.org/wiki/Work_breakdown_structure. 